\title{
22
}

\section{Resource management for service accommodation in optical networks}

\author{
Admela Jukan \\ Vienna University of Technology \\ Institute of Communication Networks \\ Gusshausstrasse 25/388, A-1040 Vienna, Austria \\ Tel. +43-1-58801-3993, Fax +43-1-5870583 \\ E-mail:admela.jukan@tuwien.ac.at
}

\begin{abstract}
In this paper, a novel approach for service accommodation in WDM networks is proposed. It is based on the classification of optical network services according to Quality-of-Service (QoS) to be guaranteed for a client network (typically, SDH or ATM). This principle is applied to node architectures and node main functions as well as to wavelength assignment. Various resource management heuristics for WDM optical networks are presented, where shifting of physical and logical resources in dynamically routed optical networks is minimised. Simulation results for an example network are presented for a demonstration of applicability of the proposed methods.
\end{abstract}

\section{Keywords}

Optical network service, QoS-routing, node architectures, resource management, blocking probability 
In multi-client optical networks, it is advantageous to classify each client according to the Quality-of-Service (QoS) to be guaranteed for that client. This is because end-to-end parameters like transmission performance, throughput, or survivability of different client networks are not required to be equal. In spite of the fact that this principle is generally applicable to multi-client networks, only wavelength division multiplexing (WDM) networks are considered in this paper.

Client networks, i.e. typically synchronous digital hierarchy (SDH) and asynchronous transfer mode (ATM) networks, are connected to optical nodes for access, where one or more WDM channels are assigned to them. The wavelength channels are circuit-switched and with a certain grade of transparency handled within an optical network. The QoS-guarantee refers to a dedicated wavelength channel, i.e. to the comprehensive client network, but not to a single end-user of that network in terms of bit patterns, audio or video quality.

In this paper, service accommodation is proposed based on classifying optical network services according to QoS-guarantee for a client network for which a service is provided. This principle will be applied to node architectures and node main functions as well as to routing and wavelength assignment. The resource management heuristics for wavelength assignments in optical networks are presented, where the number of service quality degree shifting, resource shifting as well as the number of wavelength shifting required is minimised. These methods are applied to dynamically routed optical networks. Finally, simulation results for an example network with meshed topology and various service quality degrees and heuristics are presented.

Instead of considering QoS-attributes like data patterns, video- or audio- quality, in optical networks we deal with QoS-attributes that refer to many electronically multiplexed end-users simultaneously. Therefore, "Quality-of-optical-networkService" (QoonS) was defined in (Jukan, 1997). An optical network service (ONS) handles optical signals originating from optical network clients. The most important optical network clients today are networks based on SDH and ATM.

The resource management in an optical network decides about necessary and available resources for optical network service accommodation according to the quality attributes of a certain service. The basic factors which define these quality attributes are: complexity of access interfaces, transmission performance, cross-connecting and routing functions, network supervision and management, optical network service restoration, and economic efficiency (Gruber, 1986). The performance objectives of each singular client differ. High throughput may be required for a client handling data, while video communication may require higher signal-to-noise ratio. The selection of the network route for a particular client must take into account which route is the best suited for providing the necessary quality. 
There are two possibilities how quality attributes are associated to routing (Jukan, 1997). Either routing is separated from the handling of quality attributes or the quality attributes directly take part in routing by influencing the cost of links. In both of these methods, optical network services are classified according to the quality degree required. The blocking probability is here defined as probability that for a certain service a sufficient quality cannot be provided.

NODE ARCHITECURE

Optical networks considered here are capable of providing various services for client networks, like access, transmission, routing, or alternative path searching. They consist of optical fibres, which connect optical nodes. The access for optical network clients is provided by means of client network access (CNA). Optical nodes contain QoS-selective switches realised with controllable wavelength and/or fibre switches. The functions of CNA and QoS-selective switches will be discussed in more detail. Nevertheless, other node functions like de/multiplexing, regeneration, or control, which are not discussed here, must also be realised on the basis of QoS-guarantee.

The architecture of a QoS-selective switch for an optical node is shown in Figure 1. In this figure, only the functionality of each part, but not the exclusive implementation methods is shown. A QoS-selective switch is implemented for routing of multi-channel WDM signals and for routing of single channels being demultiplexed from a WDM signal or being newly assigned from a CNA. Space switching is provided on a fibre-to-fibre basis. In Figure 1, two functional blocks for switching called Multi-Channel Shifting and Single Channel Shifting are shown. Multi-Channel Switching is capable of shifting a complete incoming WDM signal or a part of that signal containing more than one channel. This is achieved by means of Bandwidth Shifting (BS). This function can be realised with converter arrays designed for wide bandwidth conversion $(r b)$ (Iannone, 1996). Otherwise, a multi-channel signal can be bypassed $\left(r b_{0}\right)$.

Single channels can be handled as follows. They can be routed to a CNA if the affected node is the destination node $\left(r_{3}\right)$. Within the same service quality degree, single channels can be converted into another wavelength within a Wavelength Shifting (WS) array $\left(r_{l}\right)$. They can also be routed to a Quality Degree Shifting (QDS) stage for re-transmission on a new wavelength $\left(r_{2}\right)$. As previously shown, they can be bypassed, too $\left(r_{0}\right)$.

Both functions of bypassing $\left(r_{0}\right.$ and $\left.r b_{0}\right)$ might be necessary for WDM signals carrying one or more single channels of such a quality degree that need not be or can not be processed by the affected node. This is an even bigger grade-oftransparency in optical networks.

The common architecture for CNA and QDS can be seen in the bottom of Figure 1. A client network input/output is connected to a configurable point-topoint client switch (service switch). According to the service quality degree to be configured for an attached client the appropriate input/output of that switch is chosen dynamically. This input/output is furthermore connected to laser/receiver arrays. Laser/receiver arrays are supposed to be different for different quality degrees, which is in accordance with the design of commercial devices. 


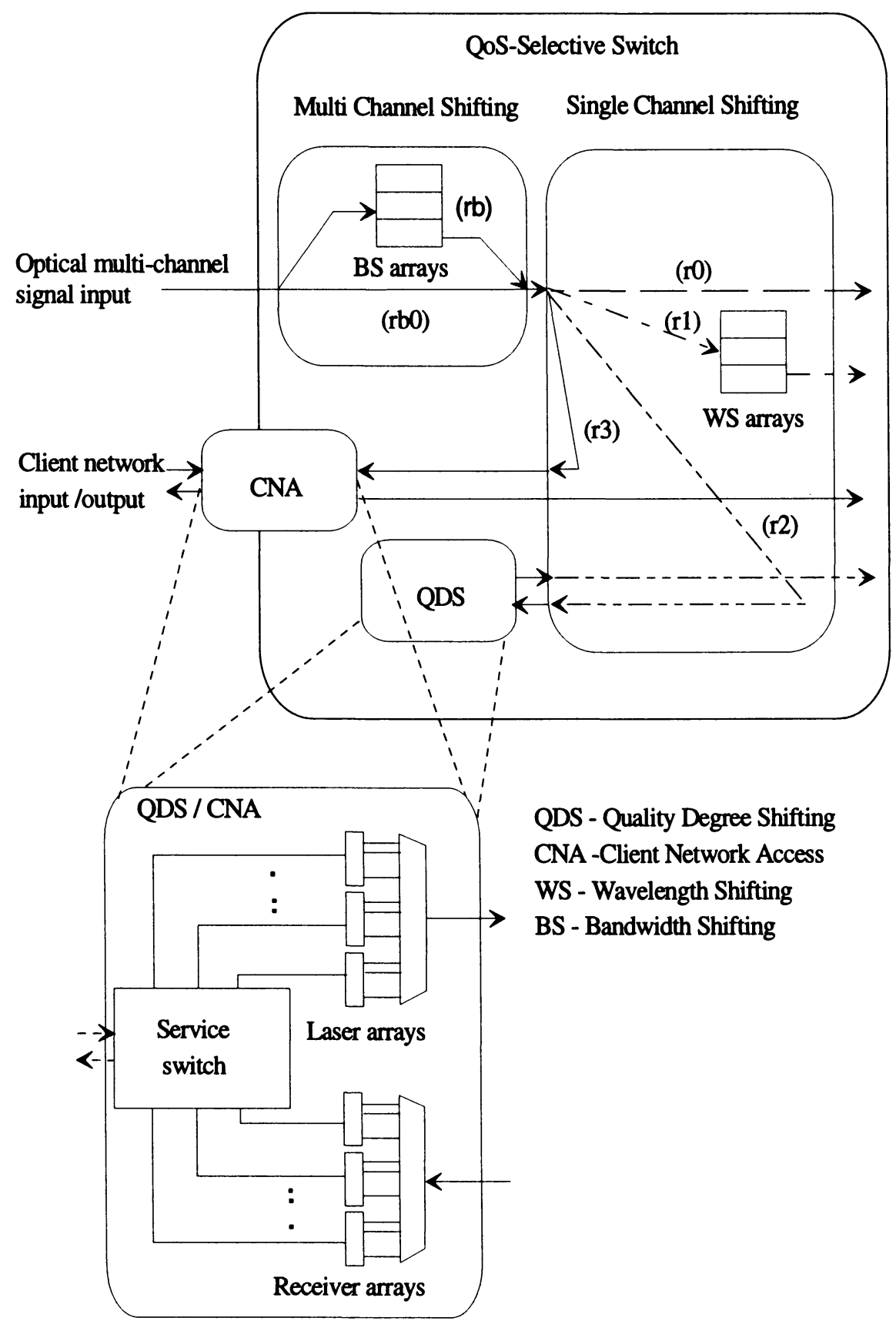

Figure 1. QoS- Selective switch with CNA and QDS architectures. Routing of optical multi-channel signals: with BS within the same service quality degree (rb) or without BS $\left(\mathrm{rb}_{0}\right)$. Routing of single optical channels: $\left(\mathrm{r}_{0}\right)$ bypassing, $\left(\mathrm{r}_{1}\right)$ all-optical conversion (wavelength shifting) within the same service quality degree, $\left(r_{2}\right)$ QDS for re-transmission on a new wavelength, and $\left(r_{3}\right)$ receiver arrays in CNA. 
With the CNA architecture as shown in Figure 1, it is not necessary to provide wavelength conversion for accessing signals, because the best transmitting /receiving wavelength can be arbitrarily chosen. This feature has large importance for wavelength continuous optical networks, where the choice of transmitting wavelength has a large impact on performance.

The QDS-architecture is almost the same as the CNA-one, only the client input/output part is missing. Here, a single channel is transparently retransmitted on a wavelength reserved for services of higher quality degree.

Summarised, service accommodation provided by the architecture as shown in Figure 1 yields the following advantages:

- Wavelength adaptation for routing is performed at CNA, which is important for optical networks without wavelength conversion where the choice of transmitting wavelength influences performance.

- Network management effort is reduced because less management and control functions are to be implemented when lower quality is to be guaranteed. Regarding economic constraints, cheaper components can be used for service classes where less quality is required.

- Different functions like wavelength shifting or network access can be partially (per service) implemented according to the particular requirements on a certain class of service which is of great importance for modular and cost effective design of optical nodes.

- Integrated components like arrays of lasers, converters, filters or receivers can be used. They are generally designed to provide a certain set of performance in defined ranges (for example, technologies for components used for handling various kinds of optical signals, like $10 \mathrm{~Gb} / \mathrm{s}$ optical transmitter and $155 \mathrm{Mb} / \mathrm{s}$ one, are different).

- With the service shifting designed with the re-transmission by means of electrical switching, an advantage might be an easy implementation of electrical regenerators. This might be unavoidable for long-haul connections.

- This architecture is suitable for operating many corporate optical networks over the same physical topology where users or group of users with different QoS requirements can be accommodated separately.

WAVELENGTH ASSIGNMENT HEURISTICS

For the wavelength assignment heuristics presented here, the following definitions are made. The primary wavelength set (PWS) for a certain service quality degree contains the wavelengths supposed to be primarily assigned before other wavelengths belonging to higher service quality degrees are taken. Resource shifting (RS) occurs when a called service is not using resources from its own PWS. Wavelength shifting (WS) refers to wavelength shifting on a linkto-link basis only between wavelengths belonging to the same PWS. Quality degree shifting (QDS) occurs on a link-to-link basis, too, and changes the current service quality degree by means of wavelength shifting into another PWS.

The basic ideas for the wavelength assignment heuristics discussed here are to minimise wavelength shifting, quality degree shifting, and/or resource shifting. 
Wavelength shifting minimisation might be necessary in order to improve transmission performance, if the cascadeability of wavelength converting is critical. When minimising quality degree shifting, less additional hardware and fewer service resources of higher quality degree are needed. Resource shifting minimisation improves network performance since each service uses its own resources.

Assume a network with $N$ nodes and $L$ links for which a wavelength set $\Lambda$ and a service set $S$ are defined as $\Lambda=\left\{\lambda_{1}, \lambda_{2}, \ldots, \lambda_{W}\right\}$ and $S=\left\{S_{l}, S_{2}, \ldots S_{P}\right\}$, respectively. The basic assumption is that each service can be provided at least by one and maximally by all wavelengths from $\Lambda$ (Jukan, 1997). Service quality degrees are defined in terms of wavelength usage. The number of wavelengths limits the maximal number of services (generally $P \leq W$ ). Assume that a particular service $S_{i} \in S, i \leq P$ may assign any wavelength from the wavelength set $W\left[S_{i}\right]=\left\{\lambda_{w 1}, \lambda_{w 2}, \ldots . \lambda_{w i}\right\}$, generally $W_{i} \leq W$. That means for every service quality degree $D_{i}$ corresponding to the service $S_{i}$ a set of pre-reserved wavelengths with $W\left[S_{j}\right]$ is defined as described above. The primary wavelength set is defined as $W_{p r i m}\left[S_{i}\right]=\left\{\lambda_{w 1}, \lambda_{w 2}, \ldots \lambda_{N p}\right\}$, generally $N_{p} \leq W_{i}$.

The problem of minimising resource-, quality degree- or wavelength shifting is here mapped to the one of finding the shortest path with least costs by using the following cost definitions. The main definitions used in equation (1)-(4) are shown in Table 1.

Cost of resource shifting $\left(C_{r s}\right)$ is defined for a service requested (called) by a client $S_{\text {call }}$ (where call is the index for quality degree $D_{\text {call }}$ ). If $S_{\text {call }}$ is using the wavelength $\lambda^{k}$ at the $k$ th link $l_{k}$ from the primary wavelength set of the higher service quality $S_{j}$ (quality degree $D_{j}$ ), then:

$$
C_{\mathrm{rs}}\left[S_{i=\text { call }}\left(\lambda^{k}\right), S_{j}\left(\lambda^{k}\right)\right]=\left\{\begin{array}{cc}
(j-i)^{*} D_{\mathrm{d}} & \text { for } i \leq j \\
\infty & \text { for } i>j
\end{array}\right.
$$

A constant $D_{d} \geq 0$ is defined a priori. The expression (1) refers to the usage of network resources with respect to the called service.

Cost of quality degree shifting $\left(C_{q d s}\right)$ is defined for the case that a service $S_{i}$, instead of using the wavelength $\lambda^{k-1}$ (used on the link $l_{k-1}$ ) on the succeeding link $l_{k}$, is re-transmitted on a $\lambda^{k}, \lambda^{k-1} \neq \lambda^{k}$, from the PWS of higher service quality degrees.

$$
\begin{aligned}
C_{q d s} & {\left[S_{j}\left(\lambda^{k-1}\right), S_{j}\left(\lambda^{k}\right)\right]=} \\
& \left|C_{r s}\left[S_{i=c a l l}\left(\lambda^{k}\right), S_{j}\left(\lambda^{k}\right)\right]-C_{r s}\left[S_{i=c a l l}\left(\lambda^{k-1}\right), S_{j}\left(\lambda^{k-1}\right)\right]\right|, \text { for } \lambda^{k-1} \neq \lambda^{k}
\end{aligned}
$$

Cost of wavelength shifting $\left(C_{w s}\right)$ from $\lambda^{k-1}$ to $\lambda^{k}$ (from the links $l_{k-1}$ and $l_{k}$, respectively) within the same primary wavelength set $W_{\text {prim }}\left[S_{i}\right], S_{i} \in S$, of any service quality degree $D_{i}$, is defined for every constant $C_{\text {conv }} \geq 0$ as: 


$$
C_{c}\left[S_{i}\left(\lambda^{k-1}\right), S_{i}\left(\lambda^{k}\right)\right]= \begin{cases}C_{c o n v} & \lambda^{k-1} \neq \lambda^{k}, \text { for } \lambda^{k-1}, \lambda^{k} \in W_{\text {prim }}\left[S_{i}\right] \\ 0 & \lambda^{k-1}=\lambda^{k}, \text { for } \lambda^{k-1}, \lambda^{k} \in W_{\text {prim }}\left[S_{i}\right] \\ \infty & \lambda^{k-1} \neq \lambda^{k}, \text { for } \lambda^{k-1}, \lambda^{k} \notin W_{\text {prim }}\left[S_{i}\right]\end{cases}
$$

Table 1 Basic definitions used in equations (1)-(4)

\begin{tabular}{|l|l|}
\hline$S_{\text {call }}\left(\lambda^{k}\right)$ & $\begin{array}{l}\text { service called for connection set-up } \\
\text { using } \lambda^{k} \text { wavelength at the link } l_{k}\end{array}$ \\
\hline$S_{h}\left(\lambda^{k}\right)$ & $\begin{array}{l}\text { highest possible service quality } \\
\text { degree allowed to use } \lambda^{k} \text { at the link } \\
l_{k}\end{array}$ \\
\hline$C^{k}\left[S_{\text {call }}\left(\lambda^{k}\right)\right]$ & $\begin{array}{l}\text { minimum cost of establishing called } \\
\text { service } S_{\text {call }} \text { over the first } k \text { network } \\
\text { links, if wavelength used at the } k \text { th } \\
\text { link is } \lambda^{k}\end{array}$ \\
\hline$C_{0}\left(l_{k}\right)$ & $\begin{array}{l}\text { basis cost of using link } l_{k} \text { for any } \\
\text { service }\end{array}$ \\
\hline$C_{w s}\left[S_{\text {call }}\left(\lambda^{k-1}\right), S_{\text {call }}\left(\lambda^{k}\right)\right]$ & $\begin{array}{l}\text { cost of wavelength shifting between } \\
\text { the links } l_{k-1} \text { and } l_{k}\end{array}$ \\
\hline$C_{q d s}\left[S_{\text {call }}\left(\lambda^{k-1}\right), S_{\text {call }}\left(\lambda^{k}\right)\right]$ & $\begin{array}{l}\text { cost of service quality degree } \\
\text { shifting between the links } l_{k-1} \text { and } l_{k}\end{array}$ \\
\hline$C_{r s}\left[S_{\text {call }}\left(\lambda^{k}\right), S_{h}\left(\lambda^{k}\right)\right]$ & cost of resource shifting at the link $l_{k}$ \\
\hline
\end{tabular}

Here, wavelength assignment heuristic returns the minimum link cost for every connection request of the service $S_{\text {call }}$ by assigning the optimal wavelength $\Lambda_{\text {opt }}$ on every link $l_{k}$ as following (Kovacevic, 1996):

$$
\begin{aligned}
C^{k}\left[S_{\text {call }}\left(\Lambda_{o p t}^{k}\right)\right] & =C^{k-1}\left[S_{\text {call }}\left(\Lambda_{o p t}^{k-1}\right)\right]+C_{w s}\left[S_{\text {call }}\left(\Lambda_{o p t}^{k-1}\right), S_{\text {call }}\left(\Lambda_{o p t}^{k}\right)\right]+ \\
& +C_{q d s}\left[S_{\text {call }}\left(\Lambda_{o p t}^{k-1}\right), S_{\text {call }}\left(\Lambda_{o p t}^{k}\right)\right]+C_{r s}\left[S_{\text {call }}\left(\Lambda_{o p t}^{k}\right), S_{h}\left(\Lambda_{o p t}^{k}\right)\right] \\
& +C_{0}\left(l_{k}\right)
\end{aligned}
$$

By applying the wavelength assignment for every link of a certain connection according to (4), the number of wavelength-, resource-, and service quality degree- shifting can be minimised. This is performed in the following steps:

1. transform the network graph with the new cost scheme by considering relevant cost functions;

2. find the shortest path between Source and Destination for the requested connection of the service requested by user;

3. minimise the number of wavelength-, resource and/or service quality degree- shifting for the path (generally, $n$-link one) found by Step 2 . 
It is not possible to optimise all of the members of the expression (4) simultaneously. This is shown in more detail in the next section.

\section{PERFORMANCE STUDY AND RESULTS}

In the performance study shown here, the following is assumed:

- three different quality degree service classes ( high - $\mathrm{H}$, medium - M, and low - L) for a meshed 40-node example network, where 8 wavelengths per link (131 links) are used for unidirectional connections;

- network load is defined as the ratio between arrival- and duration rate;

- $\quad$ wavelength primary sets per service are shown in Figure 2;

- wavelengths for lower quality services are generally allowed to use higher quality resources;

- uniform traffic distribution, Poisson service request arrivals normalised to the mean call duration, exponentially distributed call duration times, no queuing of connection requests;

- wavelength shifting is possible only within the same service quality degree, otherwise it is referred to as quality degree shifting.

w1 w2 w3 w4 w5 w6 w7 w8

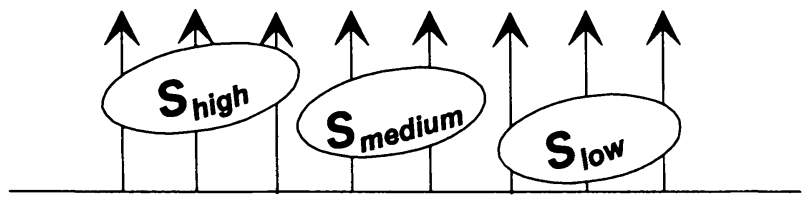

Figure 2. Primary wavelength sets for the services in the example network

For the simulation results shown, the following algorithms are applied:

- minimisation of WS only (mWS);

- minimisation of QDS (mQDS), with an additional mWS applied afterwards;

- $\quad$ minimisation of RS (mRS), with an additional mWS applied afterwards.

The blocking probability per service is shown in Figure 3. In this figure, it can be seen that the blocking probability of all three kinds of services increases with increasing network load. We modified the methods mRS and mQDS, so that after the lowest possible class of service is chosen, we still try to remain on the same wavelength even within a primary wavelength set (additional mWS). For that reason, the blocking probability is of the same order for the methods mRS and $\mathrm{mWS}$. The blocking probability of $\mathrm{H}$-services is larger for $\mathrm{mWS} / \mathrm{mRS}$ with respect to $\mathrm{mQDS}$. The contrary is true for L-services, because the usage of higher quality resources in the methods mWS and mQDS increases. Therefore, a trade-off between $\mathrm{mWS} / \mathrm{mRS}$ and $\mathrm{mQDS}$ must be found for maintaining the performance of high quality services. 


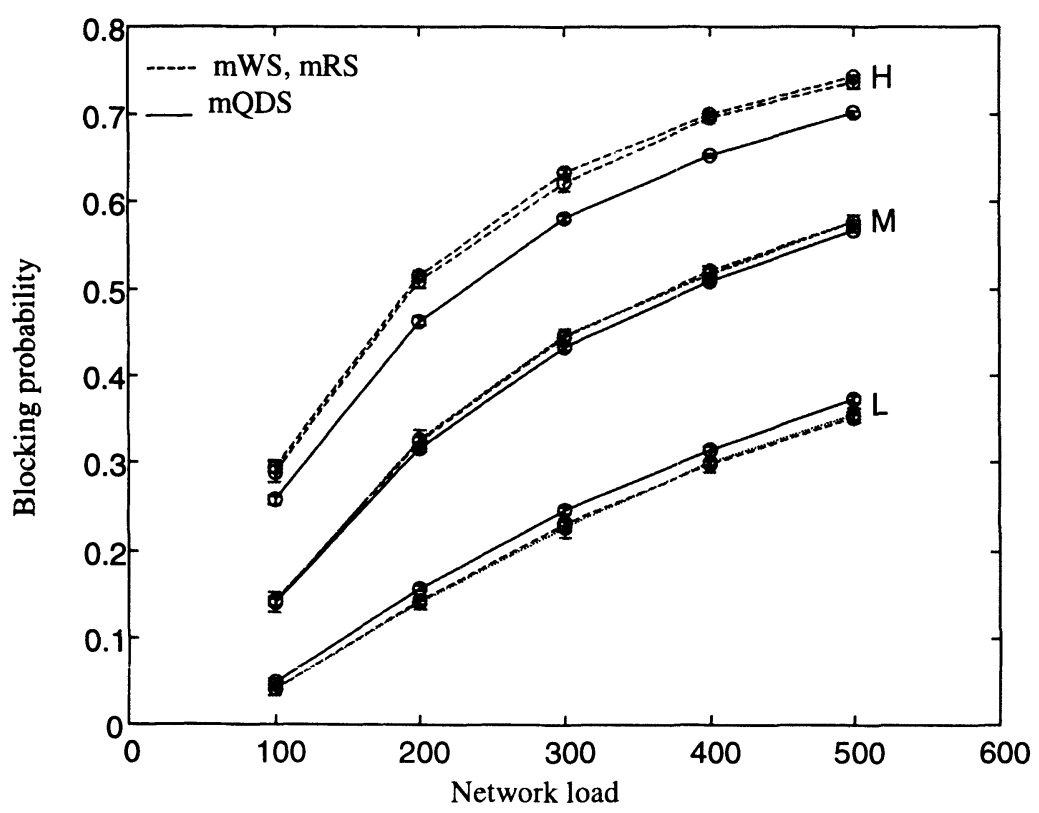

Figure 3. Blocking probability per service for each single service $(\mathrm{H}-\mathrm{high}, \mathrm{M}$ medium, L - low quality service) with the methods of minimal wavelength shifting (mWS), minimal quality degree shifting (mQDS), as well as for minimal resource shifting (mRS), calculated for an example network.

The WS-, QDS- or RS-utilisation per path length, $u_{W S, Q D S, R S}$, is defined as:

$$
u_{W S, Q D S, R S}[\%]=\frac{\text { number of necessary WS, QDS or RS }}{\text { path length in number of links }}
$$

The parameter $u$ has a practical significance, because $u_{W S}$ corresponds to the percentage of the wavelength converting devices used per link, while the parameter $u_{Q D S}$ refers to the number of wavelength re-transmitting line-cards. The parameter $u_{R S}$ refers to the percentage of resources of higher quality then necessary used for a connection. The methods of mWS, mRS and mQDS are compared for parameter $u$ (Figure 4). The wavelength conversion usage is of the same order for the methods mRS and mWS, particularly for higher loads (Figure 4a). The usage of wavelength conversion significantly decreases by applying the method mQDS. In Figure $4 \mathrm{~b}$, it can be seen that quality degree shifting is mostly performed when minimising resource- and wavelength- shifting (mRS and $\mathrm{mWS}$ ), since in both cases the same service quality degree is preferably kept. In Figure $4 \mathrm{c}$, it is shown that the method $\mathrm{mRS}$ indeed yields the minimal resource shifting, which can be used when the blocking probability of high-quality services must be improved. 


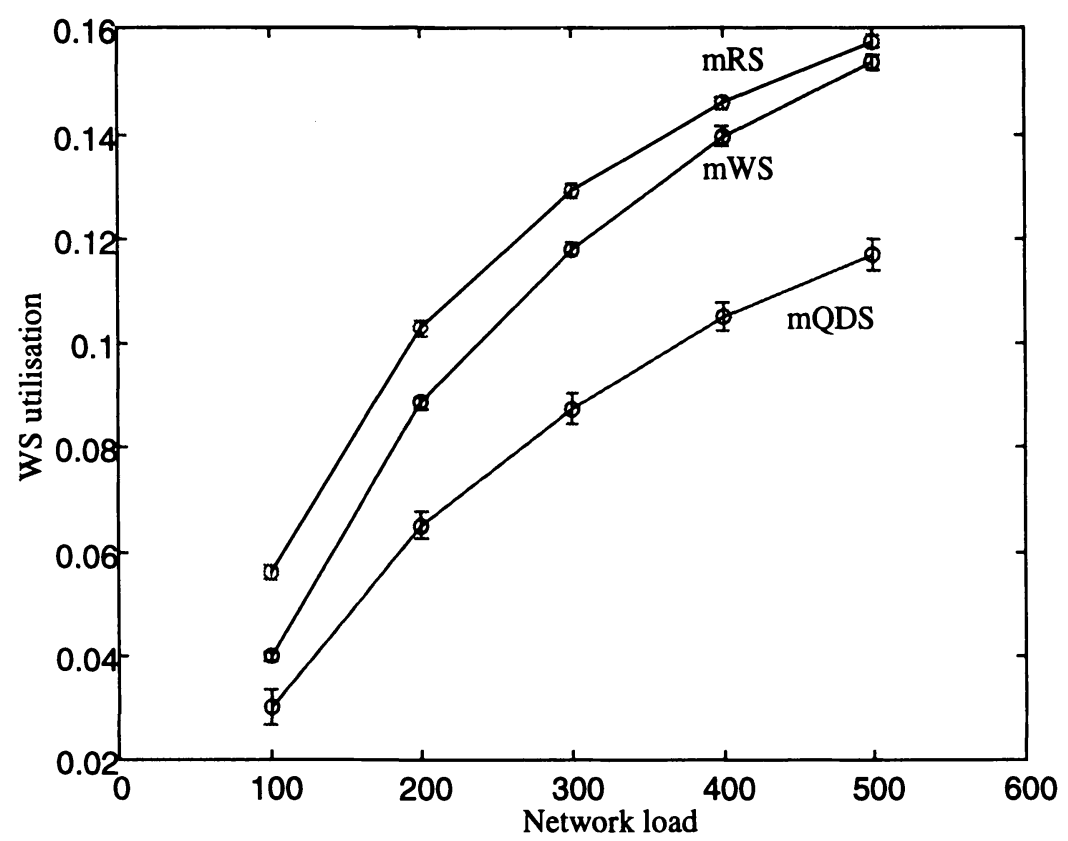

(a)

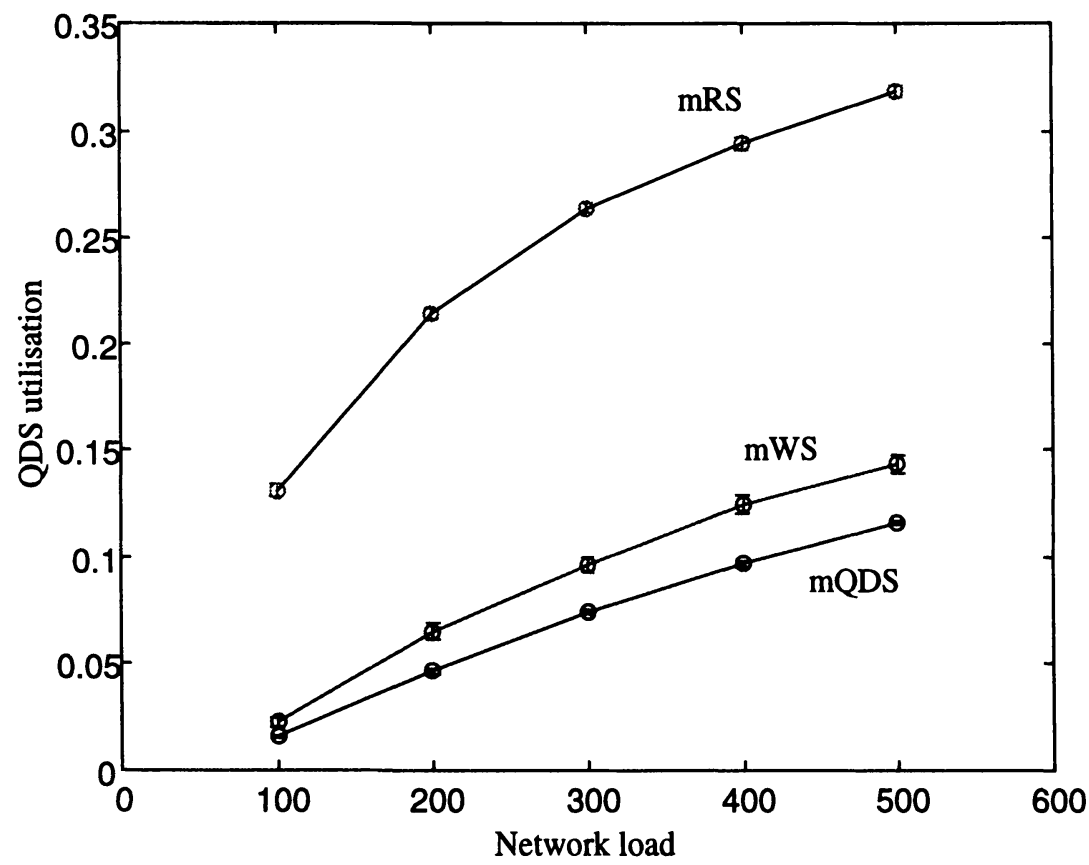

(b) 


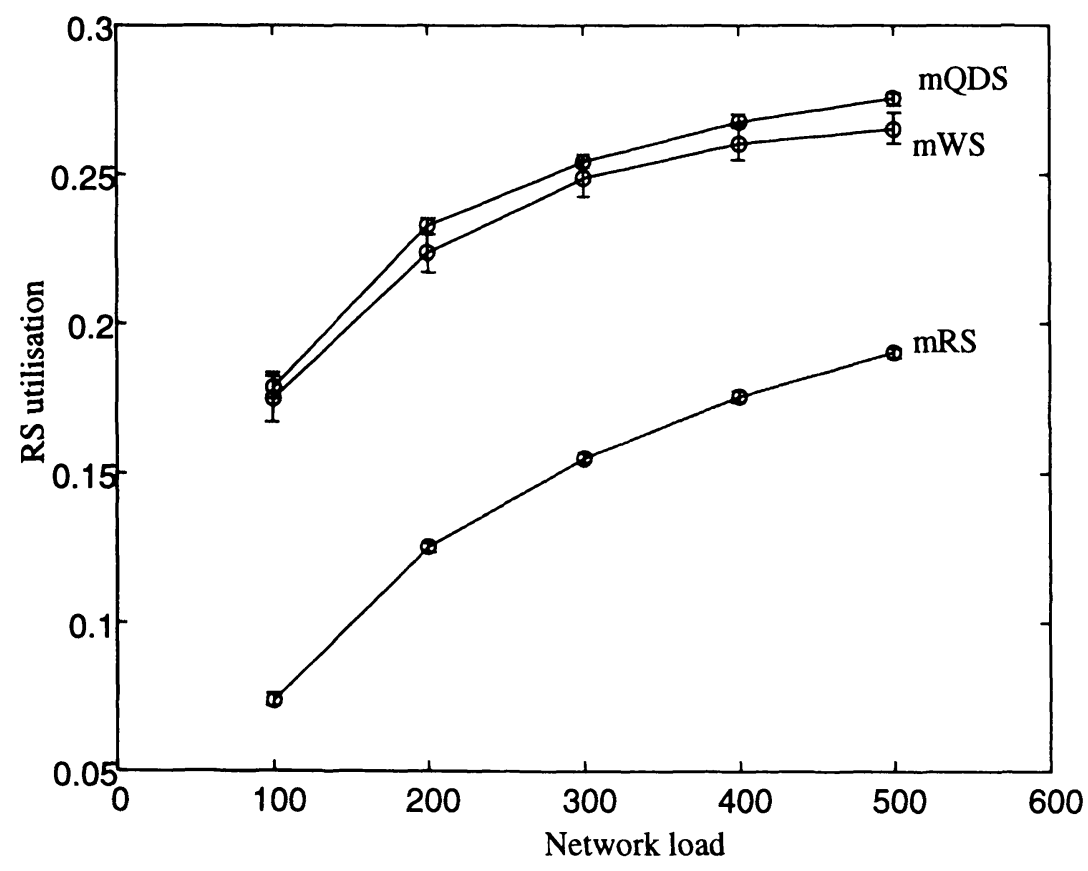

(c)

Figure 4. Utilisation of wavelength (a), quality degree (b) and resource (c) shifting for each single service ( $\mathrm{H}$ - high, $\mathrm{M}$ - medium, $\mathrm{L}$ - low quality service) for minimal wavelength shifting (mWS), quality degree shifting (mQDS), as well as for minimal resource shifting (mRS).

\section{CONCLUSION}

Based on the idea that multi-client optical networks must provide many particular services for satisfying performance requirements of each single client, a novel architecture of optical nodes, using QoS-selective switches, is proposed. The main advantages of this architecture are predictable in service-specific usage of wavelength shifting, better wavelength adaptation, reduced network management, and usage of integrated components. For this architecture, three different heuristics for wavelength assignment are proposed. With these heuristics, a route with minimal number of wavelength-, resource-, and/or quality degree- shifting is found for every service request. By means of numerical examples, it is shown that with the method of minimal resource shifting blocking probability of high-quality services can be improved. If the transparency in optical networks must be maintained or a limited number of optical converting devices or wavelength re-transmitting devices can be implemented, the minimisation methods for wavelength shifting and quality degree can be successfully implemented.

Acknowledgement: Arnold Monitzer and Harmen R. van As are acknowledged for their support and helpful discussions. 


\section{REFERENCES}

J.G. Gruber, et al (1986), Quality-of-Service in Evolving Telecommunication Networks, IEEE Journal of Selected Areas in Communication, Vol. SAC-4, No. 7, 1986, pp. 1084-1089.

E. Iannone and R. Sabella (1996), Optical Path Technologies: A Comparison Among Different Cross-Connect Architectures, IEEE Journal of Lightwave Technology, Vol. 14, No. 10, Oct. 1996, pp. 2184-2196.

A. Jukan and H.R. van As (1997), Networking based on Quality-of-opticalnetwork-Service, Proceedings NOC'97, Antwerp, Belgium, pp. 18-24.

M. Kovacevic and A. Acampora (1996), Electronic wavelength translation in optical networks, IEEE Journal of Lightwave Technology, Vol. 14, No. 6, June 1996, pp. 1161-1170.

\section{BIOGRAPHY}

Admela Jukan received the B.S. and M.S. degrees from the Faculty of Electrical Engineering and Computer Science in Zagreb and Polytechnic of Milan (CEFRIEL), respectively. She is currently working towards $\mathrm{PhD}$ degree in electrical engineering with the Institute of Communication Networks at Vienna University of Technology in Austria. Her research area focuses on QoS-based methods of resource allocation in optical networks. 